\title{
The role of epidermal growth factor-like module containing mucin-like hormone receptor 2 in human cancers
}

\author{
Michael Safaee, ${ }^{1}$ Michael E. Ivan, ${ }^{1}$ Michael C. Oh, ${ }^{1}$ Taemin Oh, ${ }^{2}$ Eli T. Sayegh, ${ }^{2}$ \\ Gurvinder Kaur, ${ }^{2}$ Matthew Z. Sun, ${ }^{1}$ Orin Bloch, ${ }^{2}$ Andrew T. Parsa ${ }^{2}$ \\ ${ }^{1}$ Department of Neurological Surgery, University of California, San Francisco, CA; ${ }^{2}$ Department \\ of Neurological Surgery, Northwestern University Feinberg School of Medicine, Chicago, IL, USA
}

\begin{abstract}
G-protein coupled receptors (GPCRs) are among the most diverse and ubiquitous proteins in all of biology. The epidermal growth factorseven span transmembrane (EGF-TM7) subfamily of adhesion GPCRs is a small subset whose members are mainly expressed on the surface of leukocytes. The EGF domains on the N-terminus add significant size to these receptors and they are considered to be among the largest members of the TM7 family. Although not all of their ligands or downstream targets have been identified, there is evidence implicating the EGF-TM7 family diverse processes such as cell adhesion, migration, inflammation, and autoimmune disease. Recent studies have identified expression of EGF-TM7 family members on human neoplasms including those of the thyroid, stomach, colon, and brain. Their pres-
\end{abstract}

Correspondence: Andrew T. Parsa, Department of Neurological Surgery, Northwestern University Feinberg School of Medicine, 676 N. St. Clair Street, Suite 2210, Chicago, IL 60611-2911, USA.

Tel.: +312.695.1801 - Fax: +312.695 .0225 .

E-mail: aparsa@nmff.org

Key words: EGF-TM7, G-protein coupled receptor, EMR2, cancer.

Contributions: MS and MEI contributed equally to this work. MS, MEI ATP, conception and design; MS, MEI, acquisition of data; MS, MEI, MCO, analysis and interpretation of data; MS, MEI, MCO, TO, ETS, GK, MZS, drafting the article; All Authors, critically revised the article and reviewed submitted version of manuscript; ATP, approved final version on behalf of all authors.

Conflict of interests: the authors declare no potential conflict of interests.

Funding: Mr. Safaee was supported by a grant from the Doris Duke Charitable Foundation. Dr. Ivan and Dr. Oh were supported by the National Research and Education Foundation from the American Association of Neurological Surgeons. Mr. Sun, Dr. Kaur, and Mr. Sayegh were supported by grants from the Howard Hughes Medical Institute. Dr. Parsa is partially supported by the Michael J. Marchese Endowed Chair in Neurological Surgery.

Received for publication: 31 December 2013.

Revision received: 14 March 2014.

Accepted for publication: 24 March 2014.

This work is licensed under a Creative Commons Attribution NonCommercial 3.0 License (CC BY-NC 3.0).

(C) Copyright M. Safaee et al., 2014

Licensee PAGEPress, Italy

Oncology Reviews 2014; 8:242

doi:10.4081/oncol.2014.242 ence on these tissues is not surprising given the ubiquity of GPCRs, but because their functional significance and pathways are not completely understood, they are of tremendous clinical and scientific interest. Current evidence suggests that expression of certain EGF-TM7 receptors is correlated with tumor grade, confers a more invasive phenotype, and increases the likelihood of metastatic disease. In this review, we will discuss the structure, function, and regulation of these receptors. We also describe the expression of these receptors in human cancers and explore their potential mechanistic significance.

\section{The adhesion G-protein coupled receptor and epidermal growth factor seven-span transmembrane subfamily}

The seven-span transmembrane (7TM or TM7) receptors are involved in a variety of signaling pathways for hormones, neurotransmitters, chemokines, ions, and sensory receptors for light, smell, and taste. They represent the largest known gene family in animals and are the target, either directly or indirectly, of most pharmaceuticals sold worldwide. ${ }^{1,2}$ The epidermal growth factor (EGF) family is the fifth most common protein family, with EGF domains present in $3 \%$ of all potential proteins. $^{3}$

The TM7 family can be classified by a variety of schemes, including division into three classes based on amino acid sequence. Class A, the rhodopsin superfamily, includes rhodopsin and other light receptors, epinephrine and adrenergic receptors, and olfactory receptors. Class B, the secretin receptor superfamily, is considerably smaller and contains receptors for gastrointestinal and other peptide hormones including growth hormone-releasing hormone, corticotropin-releasing hormone, calcitonin, and parathyroid hormone. Class $\mathrm{C}$, the metabotropic glutamate receptor superfamily, features the gamma-aminobutyric acid (GABA) receptor as well as calcium-sensing and taste receptors. The class B TM7 receptors can be further divided into three subgroups: i) B1, which contains peptide hormone receptors; ii) B2, featuring TM7 receptors with a long $\mathrm{N}$-terminal extracellular region (LNB-TM7); iii) B3, comprised of methuselah-like proteins. ${ }^{4}$ The LNB-TM7 family has over 30 members with large extracellular regions including the EGFlike domains. ${ }^{5}$ There are six members of the EGF-TM7 subfamily: i) CD97; ii) EGF-like module containing mucin-like hormone receptor 1 (EMR1); iii) EMR2; iv) EMR3; v) EMR4; and vi) EGF-TM7-latrophilinrelated protein (ETL) ${ }^{6-11}$ CD97, EMR2, and EMR3 are found on chromosome 19 at loci 19p13.1, while EMR1 and EMR4 are on 19p13.3. CD97 and EMR1 are closely linked to RFX1 and RFX2, a set of transcription factors involved in immune system function, suggesting that both families arose through multiple duplication events and eventually 
diverged. ${ }^{12,13}$ Phylogenetic analysis prompted the development of the GRAFS classification system for G-protein coupled receptors, which consists of five families: i) glutamate (G); ii) rhodopsin (R); iii) adhesion (A); iv) frizzled/taste2 (F); and v) secretin (S). ${ }^{14}$ The glutamate receptor family possesses a unique Venus fly trap N-terminus domain that allows the receptor to close around its ligand. Members of this family include the metabotropic glutamate receptors, GABA receptors, several putative taste receptors (TAS1), and a single calcium-sensing receptor. The rhodopsin family is the largest amongst $\mathrm{G}$ protein coupled receptors (GPCRs) and contains receptors for neuropeptides, chemokines, prostaglandins, biogenic amines, glycoprotein hormones, and olfactory and visual receptors.

The EGF-TM7 receptors belong to the adhesion receptor family. The adhesion GPCRs are divided into eight clusters or groups identified by Roman numerals. ${ }^{15}$ All EGF-TM7 receptors are members of Group II except for ETL, which is found in Group I. Under the GRAFS classification system, all adhesion GPCRs feature GPCR-like transmembrane domains with an $\mathrm{N}$-terminus containing an adhesion-like motif such as EGF-like repeats, mucin-like regions, or cysteine-rich motifs, and are believed to play a role in cell adhesion.,14,16 EMR2 and EMR3 are the only members of the adhesion GPCR family without mouse orthologues, further supporting the hypothesis that the EGF-TM7 subfamily arose from duplication events.

The fourth family of GPCRs contains two clusters and is referred to as the frizzled/taste2 receptor family. This group of taste receptors (TAS2) contains consensus sequences unique to the frizzled receptors and absent in the TAS1 receptors of the glutamate receptor family. ${ }^{14}$ Frizzled receptors are notable for mediating Wnt signaling and regulate cell fate and polarity during development. ${ }^{14,17,18}$ Members of the secretin receptor family are capable of binding large, typically paracrine-acting peptides. They are named after the first discovered member of the family and include the secretin receptor, glucagon receptor, calcitonin receptor, corticotropin-releasing hormone receptor, growth hormone-releasing hormone receptor, and others. ${ }^{14}$ Despite the tremendous diversity in the TM7 family, the EGF-TM7 subfamily remains unique for several reasons. First, not much is known of their downstream targets and specific ligand interactions. Furthermore, due to their fairly restricted expression profile, the functional significance of these receptors remains elusive. Finally, and perhaps most interestingly, members of this family have been detected in various malignancies, implicating them in tumor invasion, metastatic spread, and angiogenesis.

\section{Receptor structure}

Traditional GPCRs operate through a well-understood molecular cascade. Ligand binding stabilizes the receptor and stimulates a heterotrimeric guanine nucleotide-binding regulatory (G) protein to dissociate into and subunits. These subunits modulate a number of effectors including adenylyl cyclase, phospholipase, phosphodiesterase, and ion channels, which subsequently regulate the concentration of second-messengers that elicit a cellular response. It is important to note that there is evidence suggesting certain TM7 receptors may operate through G-protein-independent mechanisms. For example, in Dictyostelium, a unicellular eukaryote, cyclic adenosine monophosphate receptors (cAMP) are capable of inducing calcium influx, mitogen-activated protein kinase 1 activation, and cAMP receptor 1 phosphorylation by G-protein-independent mechanisms. ${ }^{19}$ Other TM7 receptors, such as those of the EGF-TM7 subfamily, may facilitate cell adhesion without activation of their signaling cascades, although this is less likely given the tendency of structurally similar proteins to engage the
G subunit. The EGF-TM7 receptors contain distinct regions including a cleavable signal peptide, N-terminal EGF domain region, stalk, class B TM7 region, and cytoplasmic tail (Figure 1). Most are expressed as heterodimers consisting of extracellular and TM7/cytoplasmic domains. The extracellular domains of these receptors represent some of the largest in the entire TM7 family and typically contain adjacent EGF-like domains. Like the TM7 family, the EGF domain is among the most ubiquitous in biology. EGF domains are linked to numerous physiologic processes including cell adhesion, neural development, coagulation, and fibrinolysis. ${ }^{20}$ Mutations in the EGF domains of biologically important receptors are associated with diverse human diseases. For example, mutations of the low-density lipoprotein receptor, factor IX, and fibrillin cause familial hypercholesterolemia, hemophilia B, and Marfan syndrome, respectively. ${ }^{21-23}$

The EGF-TM7 family is almost universally characterized by the presence of alternative splicing, producing isoforms with variable number and arrangement of EGF domains. ${ }^{24}$ Within this family, the EGF domains of EMR2 and CD97 demonstrate the most homology, differing by only six amino acids. ${ }^{8}$ Although EGF domains mediate the interactions between these receptors and their ligands in a calcium-dependent fashion, the intricacies of ligand binding are not fully understood. Calcium is believed to provide conformational stability to inter-domain linkages, producing EGF repeats that are highly resistant to proteolysis. ${ }^{25}$ Such properties seem ideally suited for cell-cell or cell-extracellular matrix interactions, particularly those involved in adhesion or migration. Additionally, these receptors may interact with other cell surface proteins as recent data has demonstrated heterodimerization of CD97 with lysophosphatidic acid receptor 1 (LPAR1) in prostate cancer cells. ${ }^{26}$

\section{Expression of epidermal growth factor epidermal growth factor seven-span transmembrane members}

EMR2 is expressed on monocytes, macrophages, myeloid dendritic cells, and granulocytes, but not lymphocytes. ${ }^{8,27}$ There are five EMR2 isoforms, four of which are depicted in Figure 1, that differ in their number of EGF-like domains. Interestingly, EMR2 and EMR3 are the

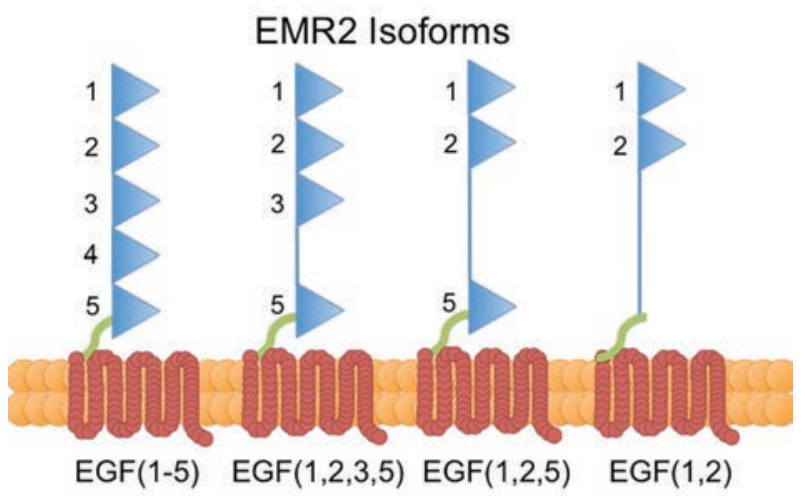

Figure 1. Isoforms of the epidermal growth factor (EGF)-like module containing mucin-like hormone receptor 2 (EMR2). These receptors feature seven-span transmembrane domains (shown in red), a mucin stalk (green), and varying numbers of EGF domains (blue triangles). The EGF-domains can be fairly large, making these proteins among the largest G-protein coupled receptors. 
only members of the EGF-TM7 family that lack mouse orthologs. EMR2 expression is highest in mature $\mathrm{CD} 16^{+}$monocytes, macrophages, and blood dendritic cell antigen- $3^{+}$myeloid dendritic cells, and lowest in granulocytes. ${ }^{27}$

\section{Functional significance of the epidermal growth factor-like module containing mucin- like hormone receptor $\mathbf{2}$ family in leukocytes}

The study of EMR2 is hampered by the lack of mouse orthologues. As the EGF domains of EMR2 demonstrate significant homology, it is not surprising that chondroitin sulfate (CS) has been identified as a ligand ${ }^{28} \mathrm{CS}$ is one of two major types of glycosaminoglycans (GAGs), the other being heparin/heparin sulfate, which differ by the composition of their amino sugars. Furthermore, there are three types of CS: i) chondroitin sulfate A (CSA); ii) chondroitin sulfate B (CSB), which includes dermatan sulfate, an isomer with variable glucuronic acid residues; iii) chondroitin sulfate $\mathrm{C}$ (CSC) ${ }^{29}$ EMR2 has been shown to bind dermatan sulfate on B cells, but does not induce signaling or cell activation..$^{30}$ This supports previous findings suggesting a role for EGF-TM7 family receptors in cell adhesion.

Functionally, EMR2 has also been implicated in autoimmune disease and neutrophil function. EMR2-positive macrophages and dendritic cells are increased in the synovium of patients with rheumatoid arthritis (RA). ${ }^{29}$ GAG expression in the inflammatory synovium and chondrocytes in RA is predominantly dermatan sulfate, while fibrotic synovium is generally comprised of CSA and CSC. ${ }^{31}$ Thus, the interaction of EMR2 with dermatan sulfate provides a plausible mechanism underlying the persistent inflammation in RA and other autoimmune diseases. Additionally, EMR2 has been implicated in neutrophil function by mediating activation and cytokine secretion in the presence of lipopolysaccharide and IL-10. ${ }^{32}$ Circulating neutrophils in patients with systemic inflammation also exhibit elevated EMR2 expression. ${ }^{33}$ Additionally, foamy macrophages in atherosclerotic vessels and splenocytes in patients with Gaucher's disease have been shown to express EMR2. ${ }^{34}$

\section{Expression of epidermal growth factor-like module containing mucin-like hormone receptor 2 in cancers}

Although once thought to be leukocyte-restricted, several studies have identified EMR2 in human neoplasms. EMR2 expression has been characterized in colorectal, breast, and brain cancers, although heterogeneity in expression patterns is detected even within these tumor types. Aust et al. found that among 81 colorectal cancer cell lines, only 8 demonstrated greater than 50\% expression of the EMR2 protein, though strong EMR2 expression was observed in a subpopulation of tumor-infiltrating macrophages ${ }^{35}$ Similarly, Davies et al. examined 5 breast cancer cell lines and found that only 2 qualified as high expressers, with EMR2 localization restricted to the peripheral membrane and perinuclear cytoplasm. ${ }^{36}$ Recently, our group examined EMR2 expression in primary glioblastoma (GBM) tissue derived from 5 patients as well as 3 established GBM cell lines, showing heterogeneous expression of EMR2 across all specimens. ${ }^{37}$ Furthermore, a cDNA microarray analysis of early-stage and advanced bladder tumors found EMR2 to be among the top-ranked genes identified. ${ }^{38}$ Unlike CD97, the most well-characterized member of the EGF-TM7 family,
EMR2 is not expressed in gastric, pancreatic, and esophageal carcinomas, which may be explained by the multiplicity of EMR2 mRNA isoforms present in single cell lines or types. ${ }^{39}$ As such, while EMR2 immunopositivity can be encountered in these neoplasms, not all tumors of any given ontology will express this protein.

\section{Functional significance of epidermal growth factor-like module containing mucin-like hormone receptor 2 in cancers}

Among patients with EMR2-positive cancers, the clinicopathologic significance of this protein varies by tumor type. In colorectal cancer, EMR2 did not significantly affect clinical parameters such as tumor stage or systemic invasion. ${ }^{35}$ However in breast cancer, EMR2 appears to have a functional role in disease aggressiveness. EMR2 is highly expressed in invasive carcinomas and a subset of ductal carcinoma in situ (DCIS), but not in normal breast epithelium. ${ }^{36}$ When comparing in situ and invasive carcinomas, EMR2 expression was significantly higher in the invasive group, suggesting that EMR2 may contribute to this invasive phenotype.$^{36}$ Interestingly, among these invasive carcinomas, increased cytoplasmic expression of EMR2 was found to correlate with a higher tumor grade, while increased nuclear expression of EMR2 correlated with prolonged relapse-free survival and overall survival..$^{36}$ In DCIS, EMR2 expression did not show any correlation with important clinical and pathologic parameters such as patient age, tumor grade, estrogen receptor positivity, tumor progression, or overall survival. ${ }^{36}$

Our group recently characterized the functional significance of EMR2 and its fellow EGF-TM7 member EMR3 in human GBM. ${ }^{37,40}$ EMR2 was found to confer an invasive phenotype in vitro, with no effect on tumor proliferation. More importantly, when stratifying tumors by their relative expression of EMR2, there is an inverse relationship between EMR2 gene expression and overall survival; patients whose tumors express high levels of EMR2 were found to have significantly shorter overall survival compared to those with tumors expressing low levels of EMR2, suggesting that EMR2 expression may represent a clinically relevant biomarker in this disease. ${ }^{37,40}$ We believe that the survival discrepancy among patients with high versus low EMR2 expressing tumors is likely related to the invasive potential of their tumors. The mechanisms underlying EMR2 expression in GBM are not well understood, nor are the possible downstream targets of the receptor, however the upregulation of EGF-TM7 family members in GBM may represent a unique genetic subtype characterized by a highly invasive phenotype, a step in tumor oncogenesis that provides a selective advantage due to its invasive potential.

Functional data suggest that EMR2 may serve as a biomarker of increased malignancy or invasive potential in breast cancer and GBM, although perhaps not in colorectal, gastric, pancreatic, and esophageal cancers (Table 1). EMR2 is also a useful cell surface marker for tumorinfiltrating macrophages and neutrophils. Taken together, EMR2 is a critically important member of the EGF-TM7 family that may one day help stratify patients by tumor phenotype, allow for more accurate prognoses, and serve as a target for novel therapeutic agents. CD97, which shares significant homology with EMR2, has been the target of therapeutic intervention through neutralizing antibodies and a proposed vaccine against its ligand CD55; perhaps a similar approach can be used for EMR2.41,42 Such inhibition of EMR2 may represent one strategy for limiting local and systemic invasion of neoplastic cells. Given that EMR2 expression has been tied to patient survival, such therapies could conceivably improve clinical outcomes for patients with EMR2positive tumors. 
Table 1. Overview of epidermal growth factor-like module containing mucin-like hormone receptor 2 in cancers.

\begin{tabular}{|c|c|c|c|}
\hline Cancer type & Expression pattern & Functional correlation & Clinical correlation \\
\hline Bladder carcinoma & $\begin{array}{l}\text { Gene expression associated with tumor } \\
\text { progression on microarray analysis }\end{array}$ & Unknown & Unknown \\
\hline Breast carcinoma & $\begin{array}{l}\text { Higher expression in invasive carcinoma compared to DCIS } \\
\text { Localized to peripheral membrane and perinuclear regions }\end{array}$ & $\begin{array}{l}\text { Positive correlation } \\
\text { with invasion }\end{array}$ & $\begin{array}{l}\text { Cytoplasmic expression } \\
\text { associated with higher } \\
\text { tumor grade } \\
\text { Nuclear expression } \\
\text { associated with longer } \\
\text { relapse-free survival and } \\
\text { overall survival }\end{array}$ \\
\hline Colorectal carcinoma & Rare expression in colorectal carcinoma & $\begin{array}{l}\text { No association with } \\
\text { migration or invasion }\end{array}$ & $\begin{array}{l}\text { No association with tumor } \\
\text { stage, differentiation, or } \\
\text { vascular/lymphatic invasion }\end{array}$ \\
\hline $\begin{array}{l}\text { Gastric and esophageal } \\
\text { carcinoma }\end{array}$ & No expression detected & Unknown & Unknown \\
\hline Glioblastoma & $\begin{array}{l}\text { Variable expression in primary cultures } \\
\text { and established cell lines }\end{array}$ & $\begin{array}{l}\text { Positive correlation } \\
\text { with invasion }\end{array}$ & $\begin{array}{l}\text { Increased gene expression } \\
\text { associated with shorter } \\
\text { overall survival }\end{array}$ \\
\hline
\end{tabular}

DCIS, ductal carcinoma in situ.

\section{Conclusions}

The association of the EGF-TM7 family member EMR2 with multiple human cancers poses interesting questions with respect to how and why neoplastic cells would upregulate their expression of leukocyte receptors. Leaning on data demonstrating the role of these proteins in adhesion, migration, invasion, and angiogenesis, it is reasonable to postulate that EMR2-positive neoplasms possess a greater capacity for infiltrative and metastatic spread. Accordingly, therapies targeting EMR2 may be useful in attenuating both local and systemic invasion, though it remains to be seen whether a clinically relevant response is achievable. One may also hypothesize that EMR2 facilitates immune evasion in cancer cells, as the presence of EGF-TM7 family members on the surface of normal leukocytes may compromise the host's ability to mount an effective immune response against EMR2-expressing cancer cells. Moving forward, recognized challenges in the study of adhesionGPCRs include their complex chimeric protein structure, limited knowledge of downstream intracellular targets, and paucity of reagents. ${ }^{43}$ Nonetheless, ongoing advances in structural and molecular biology, genome-wide analyses such as the Cancer Genome Atlas, and accumulating data on the role of the EGF-TM7 family in neoplastic states will shed new insight into these markers and aid in the development of targeted therapies for patients with aggressive and often highly invasive cancers.

\section{References}

1. Lander ES, Linton LM, Birren B, et al. Initial sequencing and analysis of the human genome. Nature 2001;409:860-921.

2. Venter JC, Adams MD, Myers EW, et al. The sequence of the human genome, Science 2001;291:1304-51.

3. Lin HH, Stacey M, Saxby C, et al. Molecular analysis of the epidermal growth factor-like short consensus repeat domain-mediated protein-protein interactions: dissection of the CD97-CD55 complex. J Biol Chem 2001;276:24160-9.

4. Stacey M, Lin HH, Gordon S, McKnight AJ. LNB-TM7, a group of seven-transmembrane proteins related to family-B G-protein-coupled receptors. Trends Biochem Sci 2000;25:284-9.
5. Fredriksson R, Gloriam DE, Hoglund PJ, et al. There exist at least 30 human G-protein-coupled receptors with long Ser/Thr-rich Ntermini. Biochem Biophys Res Commun 2003;301:725-34.

6. Gray JX, Haino M, Roth MJ, et al. CD97 is a processed, seven-transmembrane, heterodimeric receptor associated with inflammation. J Immunol 1996;157:5438-47.

7. Baud V, Chissoe SL, Viegas-Pequignot E, et al. EMR1, an unusual member in the family of hormone receptors with seven transmembrane segments. Genomics 1995;26:334-44.

8. Lin HH, Stacey M, Hamann J, et al. Human EMR2, a novel EGF-TM7 molecule on chromosome 19p13.1, is closely related to CD97. Genomics 2000;67:188-200.

9. Stacey M, Lin HH, Hilyard KL, et al. Human epidermal growth factor (EGF) module-containing mucin-like hormone receptor 3 is a new member of the EGF-TM7 family that recognizes a ligand on human macrophages and activated neutrophils. J Biol Chem 2001;276:18863-70.

10. Nechiporuk T, Urness LD, Keating MT. ETL, a novel seven-transmembrane receptor that is developmentally regulated in the heart. ETL is a member of the secretin family and belongs to the epidermal growth factor-seven-transmembrane subfamily. J Biol Chem 2001;276:4150-7.

11. Stacey M, Chang GW, Sanos SL, et al. EMR4, a novel epidermal growth factor (EGF)-TM7 molecule up-regulated in activated mouse macrophages, binds to a putative cellular ligand on B lymphoma cell line A20. J Biol Chem 2002;277:29283-93.

12. Carver EA, Hamann J, Olsen A. S, Stubbs L. Physical mapping of EMR1 and CD97 in human chromosome 19 and assignment of Cd97 to mouse chromosome 8 suggest an ancient genomic duplication. Mamm Genome 1999;10:1039-40.

13. Lin HH, Stubbs LJ, Mucenski ML. Identification and characterization of a seven transmembrane hormone receptor using differential display. Genomics 1997;41:301-8.

14. Fredriksson R, Lagerstrom MC, Lundin LG, Schioth HB. The G-protein-coupled receptors in the human genome form five main families. Phylogenetic analysis, paralogon groups, and fingerprints. Mol Pharmacol 2003;63:1256-72.

15. Bjarnadottir TK, Fredriksson R, Hoglund PJ, et al. The human and mouse repertoire of the adhesion family of G-protein-coupled receptors. Genomics 2004;84:23-33.

16. McKnight AJ, Gordon S. The EGF-TM7 family: unusual structures at 
the leukocyte surface. J Leukocyte Biol 1998;63:271-80.

17. Slusarski DC, Corces VG, Moon RT. Interaction of Wnt and a Frizzled homologue triggers G-protein-linked phosphatidylinositol signalling. Nature 1997;390:410-3.

18. Park WJ, Liu J, Adler PN. Frizzled gene expression and development of tissue polarity in the Drosophila wing. Dev Genet 1994;15:383-9.

19. Maeda M, Aubry L, Insall R, et al. Seven helix chemoattractant receptors transiently stimulate mitogen-activated protein kinase in Dictyostelium. Role of heterotrimeric G proteins. J Biol Chem 1996;271:3351-4.

20. Campbell I, Bork P. Epidermal growth factor-like domains. Curr Opin Struct Biol 1993;3:385-92.

21. Hobbs HH, Brown MS, Goldstein JL. Molecular genetics of the LDL receptor gene in familial hypercholesterolemia. Hum Mutat 1992;1:445-66.

22. Giannelli F, Green PM, Sommer SS, et al. Haemophilia B (sixth edition): a database of point mutations and short additions and deletions. Nucleic Acids Res 1996;24:103-18.

23. Dietz HC, Pyeritz RE. Mutations in the human gene for fibrillin-1 (FBN1) in the Marfan syndrome and related disorders. Hum Mol Genet 1995;4:1799-809.

24. Bjarnadottir TK, Geirardsdottir K, Ingemansson M, et al. Identification of novel splice variants of adhesion $\mathrm{G}$ protein-coupled receptors. Gene 2007;387:38-48.

25. Reinhardt DP, Ono RN, Notbohm H, et al. Mutations in calciumbinding epidermal growth factor modules render fibrillin-1 susceptible to proteolysis. A potential disease-causing mechanism in Marfan syndrome. J Biol Chem 2000;275:12339-45.

26. Ward Y, Lake R, Yin JJ, et al. LPA receptor heterodimerizes with CD97 to amplify LPA-initiated RHO-dependent signaling and invasion in prostate cancer cells. Cancer Res 2011;71:7301-11.

27. Kwakkenbos MJ, Chang GW, Lin HH, et al. The human EGF-TM7 family member EMR2 is a heterodimeric receptor expressed on myeloid cells. J Leukocyte Biol 2002;71:854-62.

28. Stacey M, Chang GW, Davies JQ, et al. The epidermal growth factor-like domains of the human EMR2 receptor mediate cell attachment through chondroitin sulfate glycosaminoglycans. Blood 2003;102:2916-24.

29. Kop EN, Kwakkenbos MJ, Teske GJ, et al. Identification of the epidermal growth factor-TM7 receptor EMR2 and its ligand dermatan sulfate in rheumatoid synovial tissue. Arthrit Rheum 2005;52:442-50.

30. Kwakkenbos MJ, Pouwels W, Matmati M, et al. Expression of the largest CD97 and EMR2 isoforms on leukocytes facilitates a specific interaction with chondroitin sulfate on B cells. J Leukocyte Biol
2005;77:112-9.

31. Kittlick PD, Bihari-Varga M, Fischer J, et al. Synovial membrane in rheumatoid arthritis: determination of glycosaminoglycans and age-dependent correlations. Exp Pathol 1980;18:197-203.

32. Chang GW, Davies JQ, Stacey M, et al. CD312, the human adhesion-GPCR EMR2, is differentially expressed during differentiation, maturation, and activation of myeloid cells, Biochem Biophys Res Commun 2007;353:133-8.

33. Chen TY, Hwang TL, Lin CY, et al. EMR2 receptor ligation modulates cytokine secretion profiles and cell survival of lipopolysaccharide-treated neutrophils. Chang Gung Med J 2011;34:468-77.

34. van Eijk M, Aust G, Brouwer MS, et al. Differential expression of the EGF-TM7 family members CD97 and EMR2 in lipid-laden macrophages in atherosclerosis, multiple sclerosis and Gaucher disease. Immun Lett 2010;129:64-71.

35. Aust G, Hamann J, Schilling N, Wobus M. Detection of alternatively spliced EMR2 mRNAs in colorectal tumor cell lines but rare expression of the molecule in colorectal adenocarcinomas. Virchows Archiv 2003;443:32-7.

36. Davies JQ, Lin, HH, Stacey M, et al. Leukocyte adhesion-GPCR EMR2 is aberrantly expressed in human breast carcinomas and is associated with patient survival. Oncol Rep 2011;25:619-27.

37. Rutkowski MJ, Sughrue ME, Kane AJ, et al. Epidermal growth factor module-containing mucin-like receptor 2 is a newly identified adhesion $\mathrm{G}$ protein-coupled receptor associated with poor overall survival and an invasive phenotype in glioblastoma. J Neurooncol 2011;105:165-71.

38. Sanchez-Carbayo M, Socci ND, Lozano JJ, et al. Gene discovery in bladder cancer progression using cDNA microarrays. Am J Pathol 2003;163:505-16.

39. Aust G, Steinert M, Schutz A, et al. CD97, but not its closely related EGF-TM7 family member EMR2, is expressed on gastric, pancreatic, and esophageal carcinomas, Am J Clin Pathol 2002;118:699-707.

40. Kane AJ, Sughrue ME, Rutkowski MJ, et al. EMR-3: a potential mediator of invasive phenotypic variation in glioblastoma and novel therapeutic target. Neuroreport 2010;21:1018-22.

41. Spendlove I, Li L, Carmichael J, Durrant LG. Decay accelerating factor (CD55): a target for cancer vaccines? Cancer Res 1999;59:2282-6.

42. de Groot DM, Vogel G, Dulos J, et al. Therapeutic antibody targeting of CD97 in experimental arthritis: the role of antigen expression, shedding, and internalization on the pharmacokinetics of anti-CD97 monoclonal antibody 1B2. J Immunol 2009;183:4127-34.

43. Lin HH. Adhesion family of $\mathrm{G}$ protein-coupled receptors and cancer. Chang Gung Med J 2012;35:15-27. 